\title{
Despliegue de siltitas columnares en el borde nororiental del Rift de Asunción en la Ciudad de Limpio
}

\section{Columnar siltstones display on the northeast border of the Asuncion Rift in the City of Limpio}

\author{
${\text { Moisés Gadea }{ }^{*} \text { (D), Selena Valdez }}^{2}$ (D), María Liz Britez ${ }^{3}$ (D), Ariel Peralta ${ }^{4}$ (D) \\ Yamil Arvez ${ }^{5}$ iD \\ ${ }^{1}$ Universidad Nacional de Asunción, Facultad de Ciencias Exactas y \\ Naturales, Departamento de Geología. San Lorenzo, Paraguay. \\ 2 Ministerio de Obras Públicas y Comunicaciones, Vice Ministerio de Minas y \\ Energía. Fernando de la Mora, Paraguay. \\ ${ }^{3}$ Consultora, Investigadora Asociada. \\ ${ }^{4}$ Universidad Nacional de Asunción, Facultad de Ciencias Exactas y \\ Naturales, Laboratorio de Sismología. San Lorenzo, Paraguay. \\ ${ }^{5}$ Consultor. Investigador Asociado.
}

Autor de correspondencia:moi7moses@yahoo.com

Resumen: Coincidente con una de las fracturas principales del rift de Asunción, se expone una pequeña elevación en forma de domo, constituida por siltitas columnares del tipo cerro Koĩ a orillas del valle del río Salado. Los prismas columnares denotan gran desarrollo de sus lados y dimensiones, en donde se presupone un cuerpo ígneo alcalino infrayacente (semejante a sus congéneres regionales) como el responsable del efecto térmico y contracción mecánica por pérdida de agua del material clástico, para el crecimiento y evolución del diaclasado columnar. Se comprueba una cantera abandonada con cierto grado depredación.

Palabras clave: areniscas y siltitas columnares, rift de Asunción, nefelinitas.

Abstract: Coincident with one of the main faults of the Asuncion rift, it is exposed a small elevation dome shaped, constituted by columnar sandstones cerro Koĩ likewise on the border of the river Salado valley. The columnar 
prisms shows great development of their sides and dimensions, where it is supposed an alkaline igneous body underneath (same as their regional congeners) as responsible of the thermic effect and mechanical contraction due to water loss off the clastic material, for the growth and evolution of the columnar jointing. It is ascertained an abandoned quarry certainly depredated.

Keywords: columnar sandstones and siltstones, rift of Asunción, nephelinites.

\section{INTRODUCCIÓN}

\section{Las Areniscas Columnares. Antecedentes}

Eckel(1) había reportado la presencia de pequeños cuerpos de areniscas arcósicas con disyunción columnar en Areguá y Luque. Las denominó pseudotraquitas, y argumentó que se requerían mayores estudios para la compresión de su origen. No obstante, a modo de conjetura, dijo que podría tratarse de un derrame, sill, intrusivo clástico; o de un metamorfismo de rocas sedimentarias por la presencia de un cuerpo ígneo cercano.

La siguiente descripción reportada, la realizó $\operatorname{Miraglia}^{(2)}$, cuando denominó areniscas metamorfoseadas a estas rocas con disyunciones columnares, y propuso una asociación entre las mencionadas rocas y las vulcanitas cenozoicas.

Por las facilidades de acceso, cercanía y los paisajes únicos, las areniscas columnares en el Departamento Central fueron estudiadas por diversos autores (Arribas y Latorre (1982)(3); Spinzi (1986)(4); Velázquez et al. $(2008)^{(5)}$ y Gomes et al. (2014)(6) , especialmente en Areguá, en donde se encuentran sus connotados exponentes orográficos tales como los cerros Koĩ y Chororĩ.

\section{Distribución Geográfica Conocida en Paraguay}

En el Paraguay existen varias zonas de manifestaciones de areniscas columnares: aquellas que se encuentran en poblados cercanos a Asunción, en un contexto estructural de rift; en y los alrededores de la cordillera del Ybyturuzú en el departamento de Guairá; una exposición en la cordillera de los Altos en Escobar, de las cuales poco se conoce, y otra más alejada hacia el sur, en San Ignacio - Misiones. 
En lo referente a exposiciones en las cercanías de Asunción, estas litologías también se manifiestan en algunos parajes restringidos en el bajo Chaco, tales como en Benjamín Aceval y Villa Hayes, hacia el norte; y en el extremo sur, en el cerro Patiño, Itauguá; siempre asociados con los cuerpos magmáticos de la Provincia Alcalina de Asunción(7) y a zonas de fracturas (Figura 1).

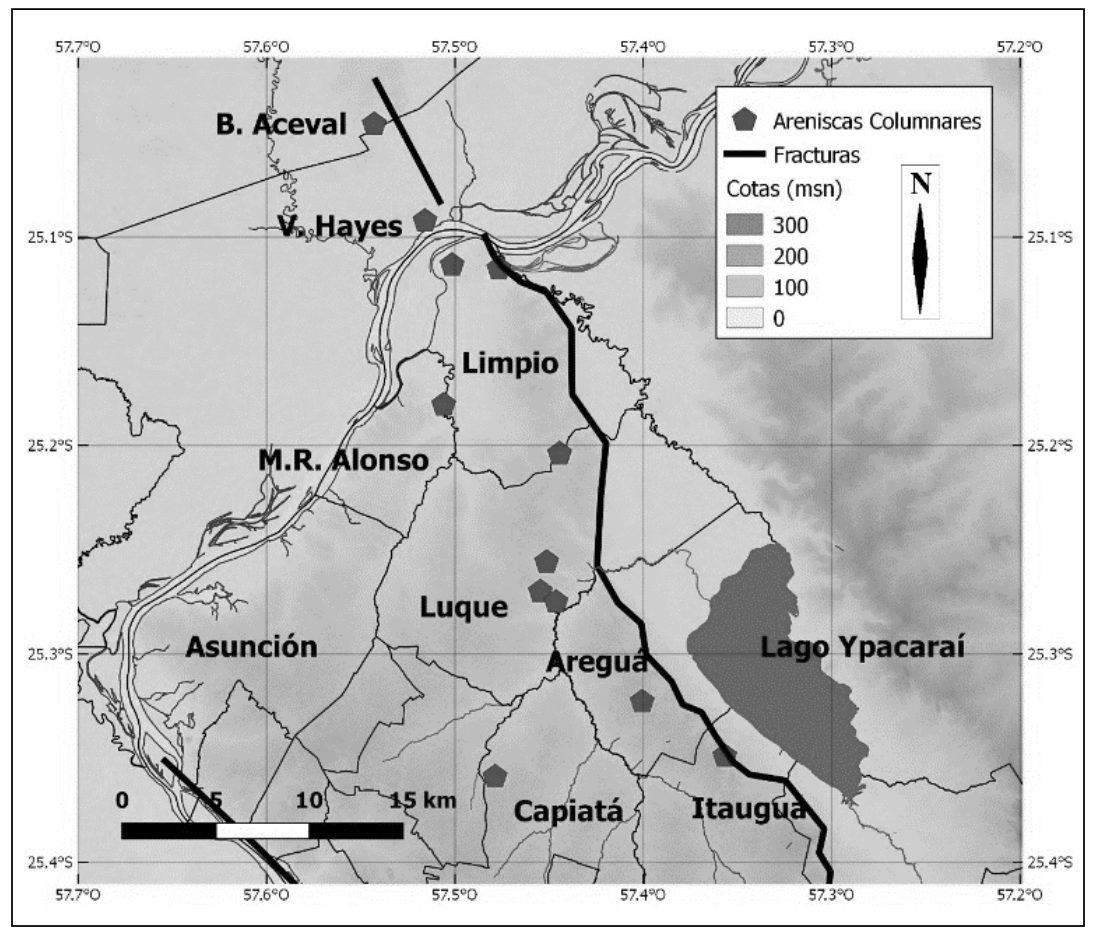

Figura 1. Manifestaciones de disyunción columnar en materiales clásticos en el departamento Central y alrededores. Elaboración propia (Gadea, 2019).

Mediante dataciones radiométricas de las nefelinitas y asociados $(7,8)$, resulta posible inferir aproximadamente las edades del desarrollo de los diaclasados columnares en los materiales clásticos previamente depósitados en un ambiente fluvial(9); debido a que la solidificación de las rocas ígneas cenozoicas y el diaclasado columnar en los materiales clásticos, si no coinciden como eventos petrofísicos, son muy cercanos entre sí en el tiempo de sus respectivos orígenes.

Estas areniscas diaclasadas que aparecen en los poblados aledaños a Asunción se relacionan genéticamente con los eventos tectónicos del tipo 
rifting, que tuvieron su inicio en el Jurásico Superior - Cretácico Inferior(11).

Para los procesos de ascenso de los magmas alcalinos los dislocamientos anteriormente originados durante los procesos de rifting sirvieron como conductos de tal forma a que los magmas se aproximen a la superficie a través de la corteza terrestre ${ }^{(10)}$.

El citado magmatismo tuvo lugar durante el Terciario Inferior, en el lapso de 61 - 39 millones de años ${ }^{(8)}$. Este pulso tectónico y magmático durante el Cenozoico es conocido como la Reactivación del Ciclo Andino(12,13).

El efecto hidrotermal de estos magmatismos produjo un recristalizado de las areniscas encajantes ${ }^{(4)}$, y el diaclasado columnar en las mismas se relaciona con su enfriamiento y contracción termomecánica por pérdida de agua(5).

Es relevante mencionar, que no en todos los sitios de manifestación de las areniscas columnares se comprueba directamente la presencia de un cuerpo ígneo asociado, como por ejemplo las de Luque. Sin embargo, no se concibe el desarrollo de los prismas columnares en areniscas sin el efecto calorífico e hidrotermal de un foco ígneo(9), por lo cual, aunque no se expongan, se los asume inmediatos.

\section{METODOLOGÍA}

Se ha realizado un recorrido exploratorio en la zona, y se ha comprobado la presencia de siltitas columnares, semejantes a las reportadas anteriormente en otros parajes. Cabe señalar que el hallazgo de esta exposición de rocas ha sido fortuito.

Se describió in situ la morfología y el emplazamiento de la cantera. Así también, el aspecto textural macroscópico de la roca. Se ha georreferenciado el lugar con un instrumento digital Garmin GPSmap76CSx, y posteriormente se elaboró un mapa (Figura 1) de despliegues de areniscas columnares en la región. Para el efecto, se trabajó con el programa QGis v 2.18 (Las Palmas).

Luego, se ha recurrido a los trabajos publicados anteriormente en lo referente al tema, para conocer los aspectos genéticos y geográficos $\mathrm{y}$ realizar comparaciones e interpretaciones. 
Rev. Soc. cient. Parag. 2020;25(2):121-130

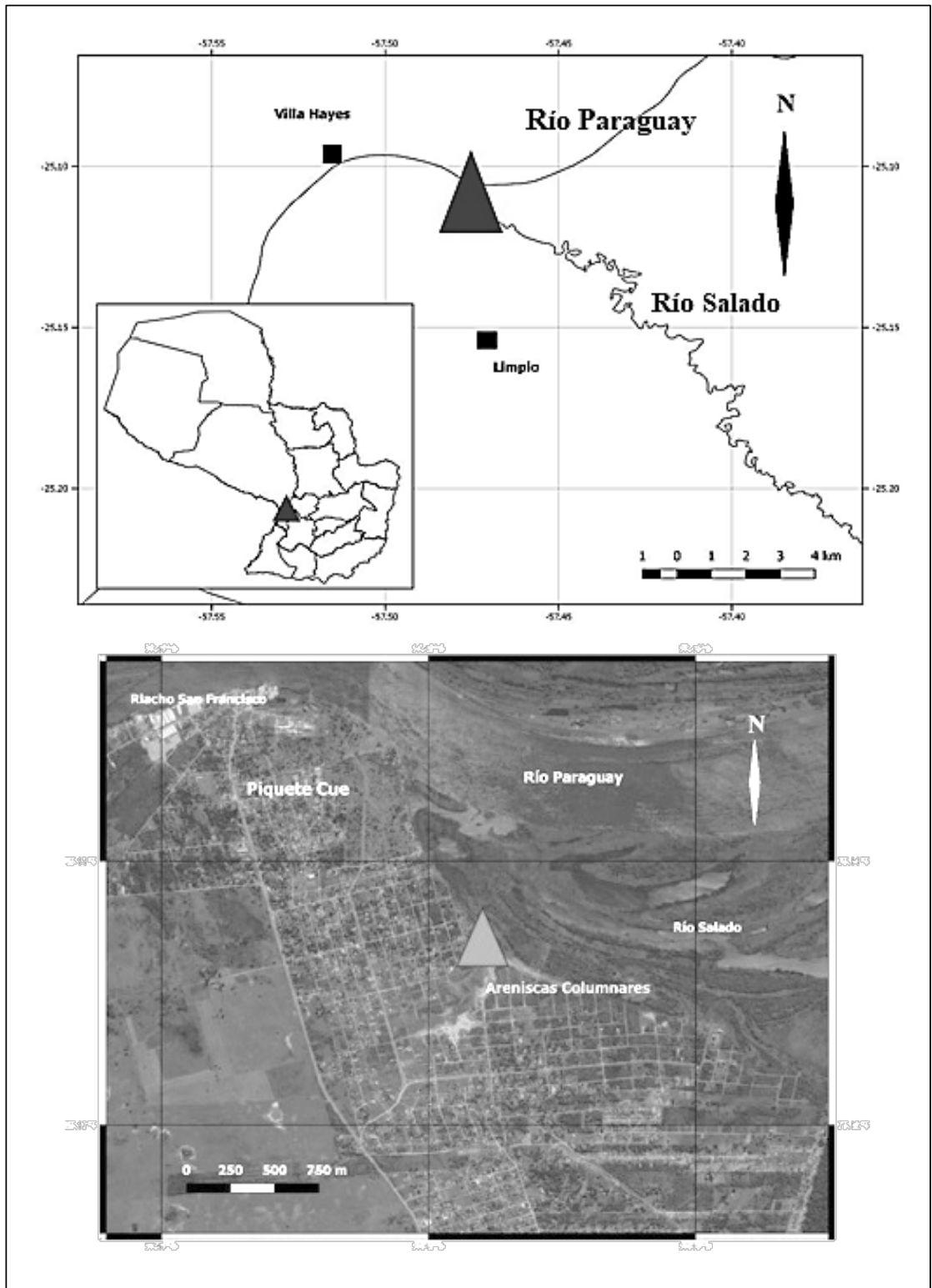

Figura 2. a. Localización de la zona de investigación. Elaboración propia (Gadea, 2019). b. Zona de confluencia del río Salado con el río Paraguay. Elaboración propia (Gadea, 2019).

\section{Geografía}

Gadea M, Valdez S, Britez ML, Peranta A, Arvez Y. Despliegue de SiltitasColumnares en el Borde Nororiental del Rift de Asunción en la Ciudad de Limpio. 
El lugar de ocurrencia se sitúa en el barrio Inmobiliaria, en el asentamiento Caacupe mí de Limpio, en el extremo Nororiental del departamento Central, según las coordenadas $-25.114927^{\circ} ;-57.476769^{\circ}$ (Figura 2a y 2b).

\section{RESULTADOS}

Una de las ciudades con mayor número de exposiciones de rocas sedimentarias con disyunción columnar según se ha comprobado, es la de Limpio, y que hasta el presente no se ha reportado oficialmente su totalidad de ocurrencias.

En el borde oriental del conjunto de pequeñas elevaciones en la zona de Piquete Cue, antecediendo inmediatamente a las planicies del valle de Ypacaraí, y a pocos metros del río Salado en su desembocadura al río Paraguay, se localiza esta manifestación de siltitas columnares (Figura $2 b$ y Figura 3).

Este despliegue litológico coincide con una de las principales fracturas del rift de Asunción en su porción nororiental.

El control tectónico y magmático de estas areniscas se comprueba, desde el cerro Patiño en Itauguá, hasta las inmediaciones de Benjamín Aceval en el bajo Chaco (Figura 1), por lo cual, no sería de extrañar el hallazgo de este tipo de afloramientos en cualquier punto intermedio en las cercanías de este segmento, determinado por la disposición geográfica de la fractura oriental del rift de Asunción, como es el caso de estas siltitas reportadas en el presentetrabajo.

Estas areniscas en Limpio, se disponen en forma de domo que sobresale en el paisaje circundante, con una superficie de exposición de no muchos metros cuadrados y se trata de un remanente erosionado.

Se propone que la forma de domo se debe a la presencia de un cuerpo ígneo del tipo dique en subsuperficie, lo cual debería comprobarse con otros trabajos exploratorios, porque nofue posible identificar rocas ígneas o algún indicio directo de ellas durante la exploración en el sitio. 
Rev. Soc. cient. Parag. 2020;25(2):121-130

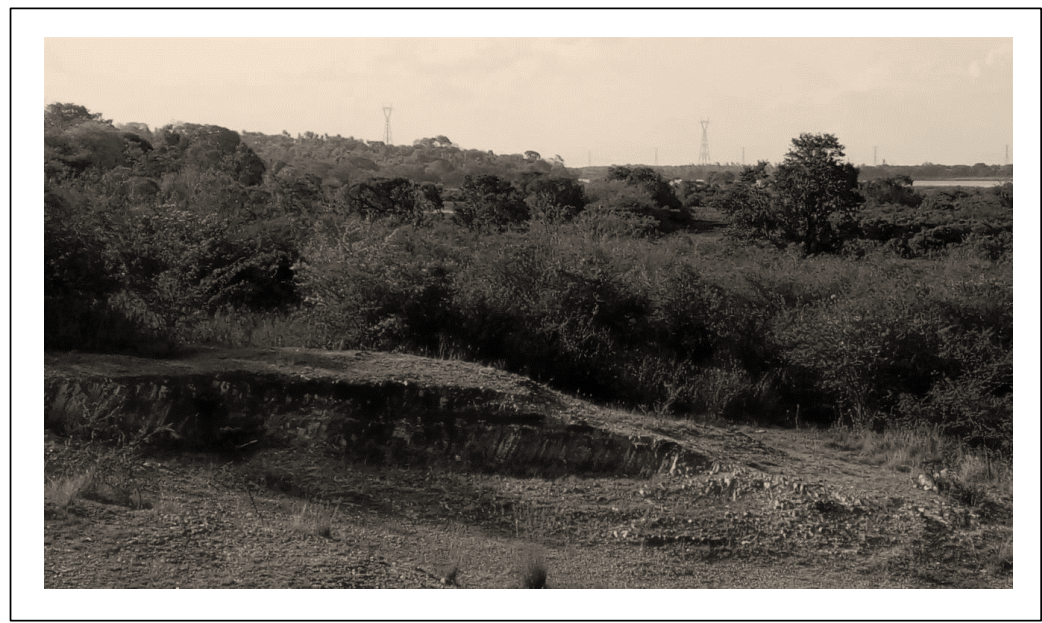

Figura 3. Vista en dirección NO-SE. Se observa en río Paraguay y las lomas de Piquete.

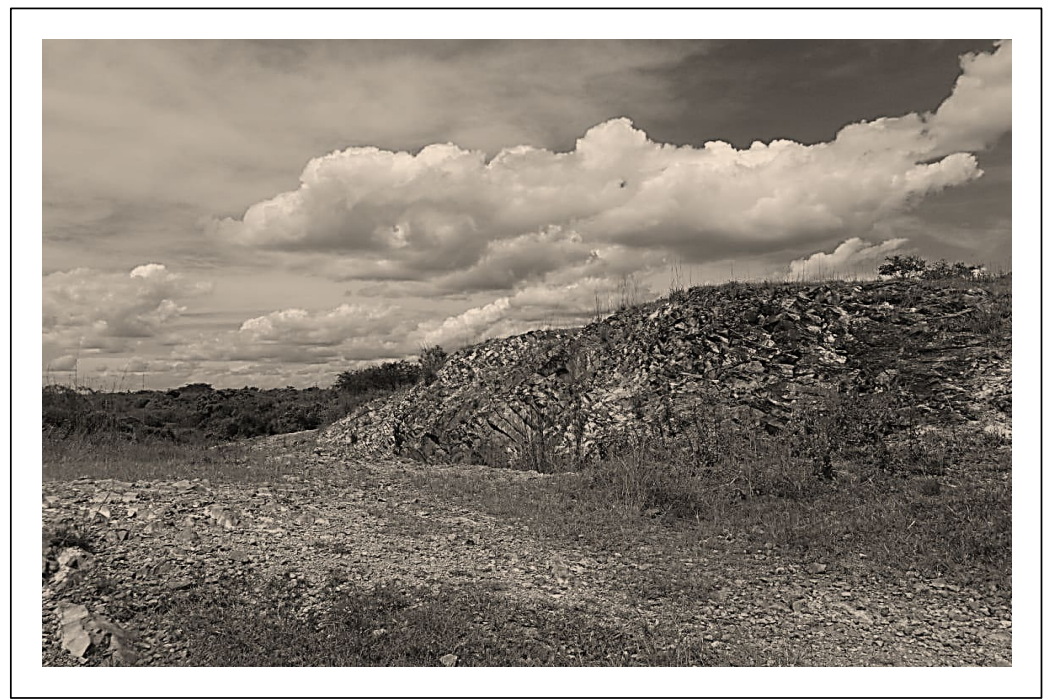

Figura 4. Exposición en forma de domo y frente de cantera.

La planicie del valle de Ypacaraí se inicia inmediatamente posterior a la caída en su flanco lateral hacia el noreste, por lo cual se reconoce posicionamiento del domo en la interface con el Alto de Asunción. Es notorio que se trata de una cantera abandonada en donde se verifica cierto grado de depredación (Figura 4). 
El material original afectado por los efectos térmicos se describe como clástico, limo-arenoso, subordinadamente arcilloso, muy compacto, de color blanco y con manchas rojas aleatorias en proporciones semejantes. Estas manchas rojas señalan una presencia de precipitados químicos en forma de hematitas en ambientes oxidantes, introducidos sindiagenéticamente y distribuidos en su matriz por diferenciación, que posteriormente fueron lavadas, lo cual señala un empobrecimiento delos óxidos en estas rocas.

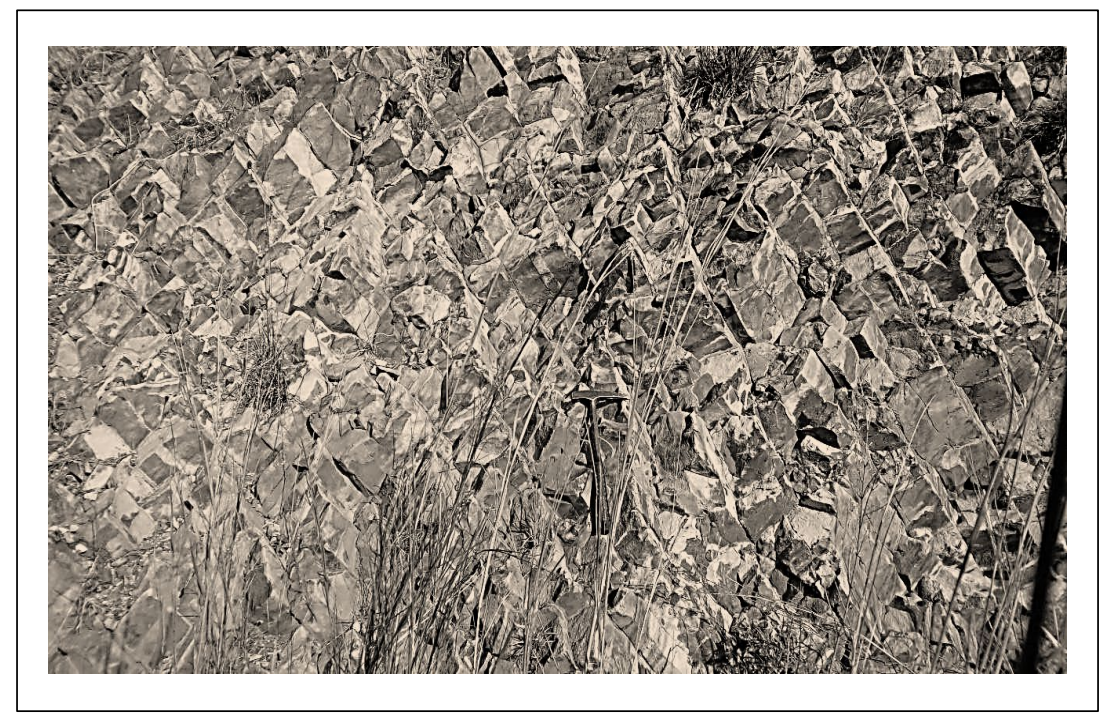

Figura 5. Prismas columnares con gran desarrollo en forma y tamaño.

Los prismas son hexagonales, pentagonales y tetragonales, con casi 15 centímetros de sus lados como predominio. Se inclinan aproximadamente a $60^{\circ}$ de la horizontal hacia el noreste.

Habiendo recorrido varios lugares con manifestaciones de rocas con disyunción columnar, se puede afirmar que éste afloramiento es uno de los que presenta mayor desarrollo de sus prismas en cuanto a sus tamaños (Figura 5), comparables a las notables areniscas columnares de Maramburé en Luque.

\section{CONCLUSIONES}

Se reporta una nueva ocurrencia de siltitas columnares en la ciudad de Limpio. 
Estas siltitas columnares se localizan en la frontera del valle de Ypacaraí en la cuenca del río Salado y las elevaciones de Piquete Cue que pertenecen al Alto de Asunción, cuya diferencia de cotas se debe por dislocamientos.

Este emplazamiento es coherente en su génesis geológico y disposición con los conocidos y reportados anteriormente en la zona de Areguá. El domo de siltitas columnares se posiciona en una de las zonas de fracturas del rift de Asunción, por donde ascendieron los magmas alcalinos afectando a las rocas clásticas. Esto presupone un control tectónico y magmático para el desarrollo de estos diaclasados columnares.

Aunque no se ha reconocido en superfice, se estima la presencia de un cuerpo ígneo por debajo de este domo de siltitas columnares. Se ha comprobado una correlación entre la existencia de materiales clásticos con prismas columnares asociados con cuerpos ígneos en los demás sitios.

Se trata de una pequeña cantera abandonada, con cierto grado de depredación. De no ser por el destape, muchas características litológicas del lugar no podrían haber sido apreciadas.

\section{REFERENCIAS BIBLIOGRÁFICAS}

1. Eckel E. Geology and Mineral Resources of Paraguay - A Reconnaissance. Geological Survey Professional Paper 327. United States Government Printing Office. Washington; 1959.

2. Miraglia L. Vulcanismo Postpliocenico Andino a lo largo del Río Paraguay. Soc. C. Par. Conferencias, Asunción, 1962; 73-80 pp.

3. Arribas A, Latorre C. El Origen de la Disyunción Columnar en Areniscas: caso de las Cuarciarenitas de Areguá (República del Paraguay). Madrid, Tecniterrae; 1982.

4. Dirección de Recursos Minerales (MOPC). Meta Areniscas Columnares del Cerro Koi y Chorori - Informe Técnico, Angel Spinzi, Asunción; 1986.

5. Velázquez V, Giannini P, Riccomini C, Sallun A, Hachiro J, Gomes C. The columnar joints in the Patiño Formation sandstones, Eastern Paraguay: a dynamic interaction between dyke intrusion, quartz dissolution and cooling-induced fractures. Epis., 2008;31(3):302-308. 
6. Gomes CB, Castillo AM, Gadea M, Comin-Chiaramonti P. Polygonal Jointing in Sandstones from Easter Paraguay. Ser. Corr. Geol., 2014,30(1):13-23.

7. Velázquez V. Provincia Alcalina Central, Paraguai Centro-Oriental: Aspectos Tectónicos, Petrográficos e Geocronológicos. Tesis de Maestría. Universidad de São Paulo, São Paulo; 1992.

8. Comin-Chiaramonti P, Civetta L, Petrini R, Piccirillo E, Bellieni G, Censi P, Bitschene P, Demarchi G, De Min A, Gomes C, Castillo A, Velázquez J. Terciary Nephelinitic Magmatism in Easter Paraguay: Petrology, Sr-Nd isotopes and genetic relationships with associated spinel, peridotitexenoliths. Eur. J.Mineral. 1991;3:507-525.

9. Genez L. Caracterización del Ambiente de Sedimentación de las Areniscas Columnares del Cerro Koi de Areguá, Paraguay Oriental. Tesis para la obtención de Grado, Mención Geología. Facultad de Ciencias Exactas y Naturales - UNA. San Lorenzo; 2014.

10. Velázquez V, Riccomini C, Gomes C, Figueredo L, Figueredo C. Relações Tectônicas do Magmatismo Alcalino do Paraguai Oriental. Rev. IG. 1998;19(1/2):43-49.

11.Riccomini C, Velázquez V, Barros G. Cenozoic Lithospheric Faulting in the Asunción Rift, Eastern Paraguay. Jour. S. Amer. Earth Scien. 2001;14:625-630.

12. Ministerio de Defensa Nacional. Comisión Nacional de Desarrollo Regional. Proyecto PAR 83/005, Mapa Geológico del Paraguay. Texto Explicativo. Asunción; 1986.

13. Ministerio de Obras Públicas y Comunicaciones. Dirección de Recursos Minerales, Instituto Federal de Geociencias y Recursos Naturales (BGR). Hoja Caacupé 5470. Texto Explicativo. Adela Dionisi, Asunción; 1999. 